\title{
Quantifying Gut Microbiome in Rats with Adenine-Induced Chronic Kidney Disease and the Effect of Treatment with Gum Arabic
}

Asma Al-Baniali ${ }^{*}$, Banan Shoair ${ }^{*}$, Maha Al-Asmakh ${ }^{1}$, Ola Al-Jammal ${ }^{2}$, Mohammad Sohail ${ }^{2}$ ${ }^{1}$ Biomedical Sciences Department, College of Health Sciences, Qatar University, ${ }^{2}$ Biomedical Research Center, Qatar University, Doha, Qatar

\section{ABSTRACT}

Chronic kidney disease (CKD) affects $\sim 10 \%$ of Qatar's population. Recently, dysbiosis in the gut microbiome has been associated with CKD. It is not understood whether CKD affects the gut microbiome or the dysbiotic gut microbiome leads to CKD. Gum Arabic (GA) is a fiber-rich dietary substance that has a potential to enhance the gut microbiome, therefore it could treat CKD. The Aim of this study is to quantify the gut microbiome in CKD rats and to evaluate the GA as a potential treatment for CKD.

\section{INTRODUCTION}

- The gut microbiome composition is shaped in a way that enables it to maintain a symbiotic relationship with the host body.

- Dysbiosis of the gut microbiome could be associated with a variety of diseases, which includes CKD.

- CKD is an incurable, progressive disease that is associated with systematic symptoms, such as inflammation.

- Although incurable, CKD can be manageable with different interventions, and GA is considered a potential treatment, since it could affect the gut microbiome.

\section{METHODOLOGY}

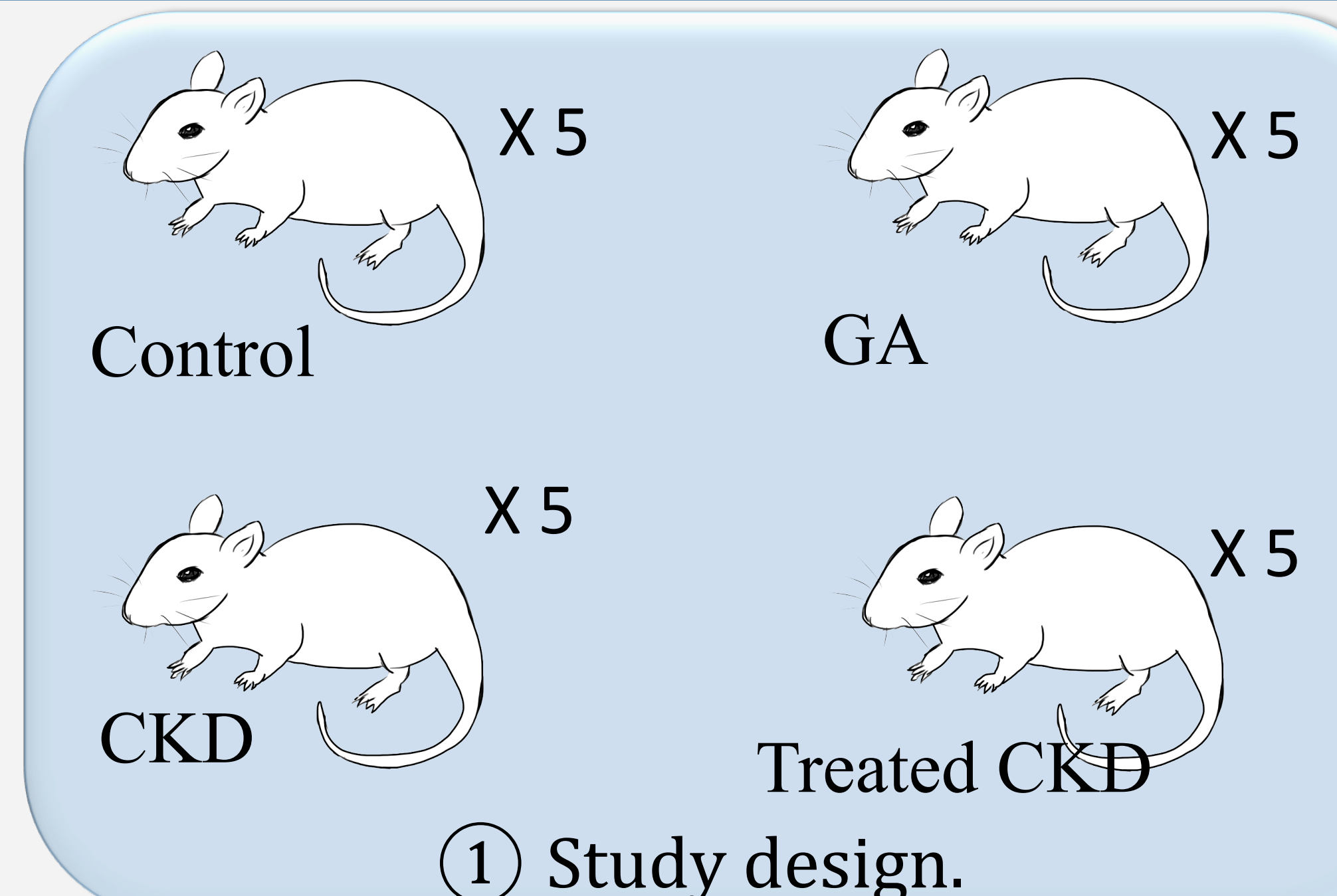

(2) Sample collection from colon, caecum and duodenum.

\section{$\sqrt{ }$}

(3) DNA extraction

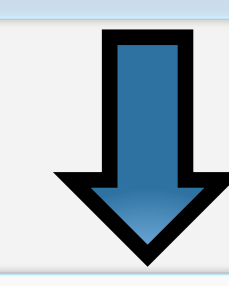

(4) DNA library preparation.

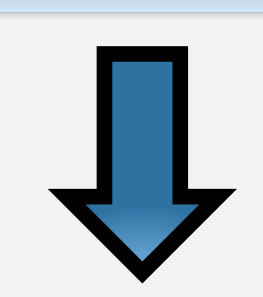

(5) Illumina MiSeq loading for NGS.

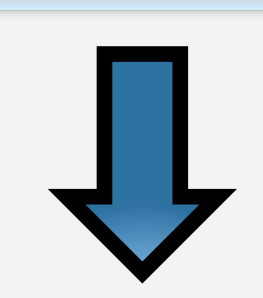

(6) Data analysis using QIIME2.

\section{RESULTS}

Fig1

Gut microbial composition at the phylum
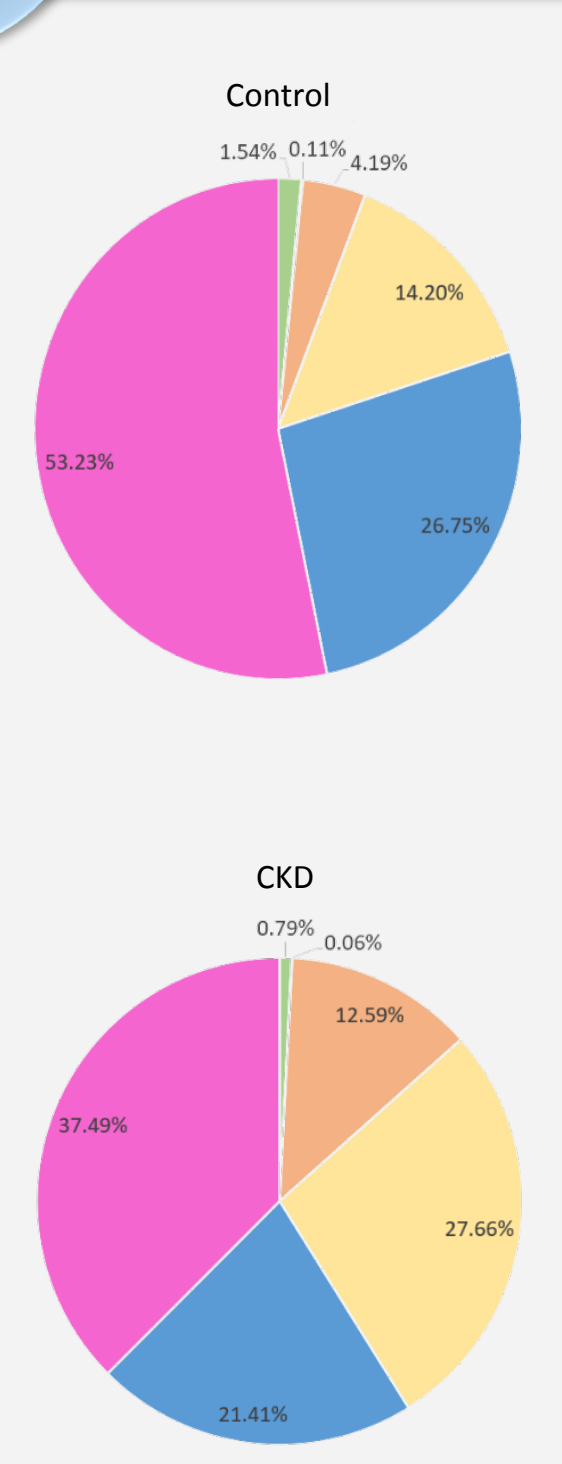

Fig2

Alpha diversity in the gut among the study groups.

Alpha diversity analysis shows that the diversity of the microbial populations in the gut of control rats is significantly higher than in the gut of CKD rats ( $p$ value $<0.05$ )

Fig3

Alpha diversity at different sites among the study groups.

Fig4

Beta diversity analysis in the colon.

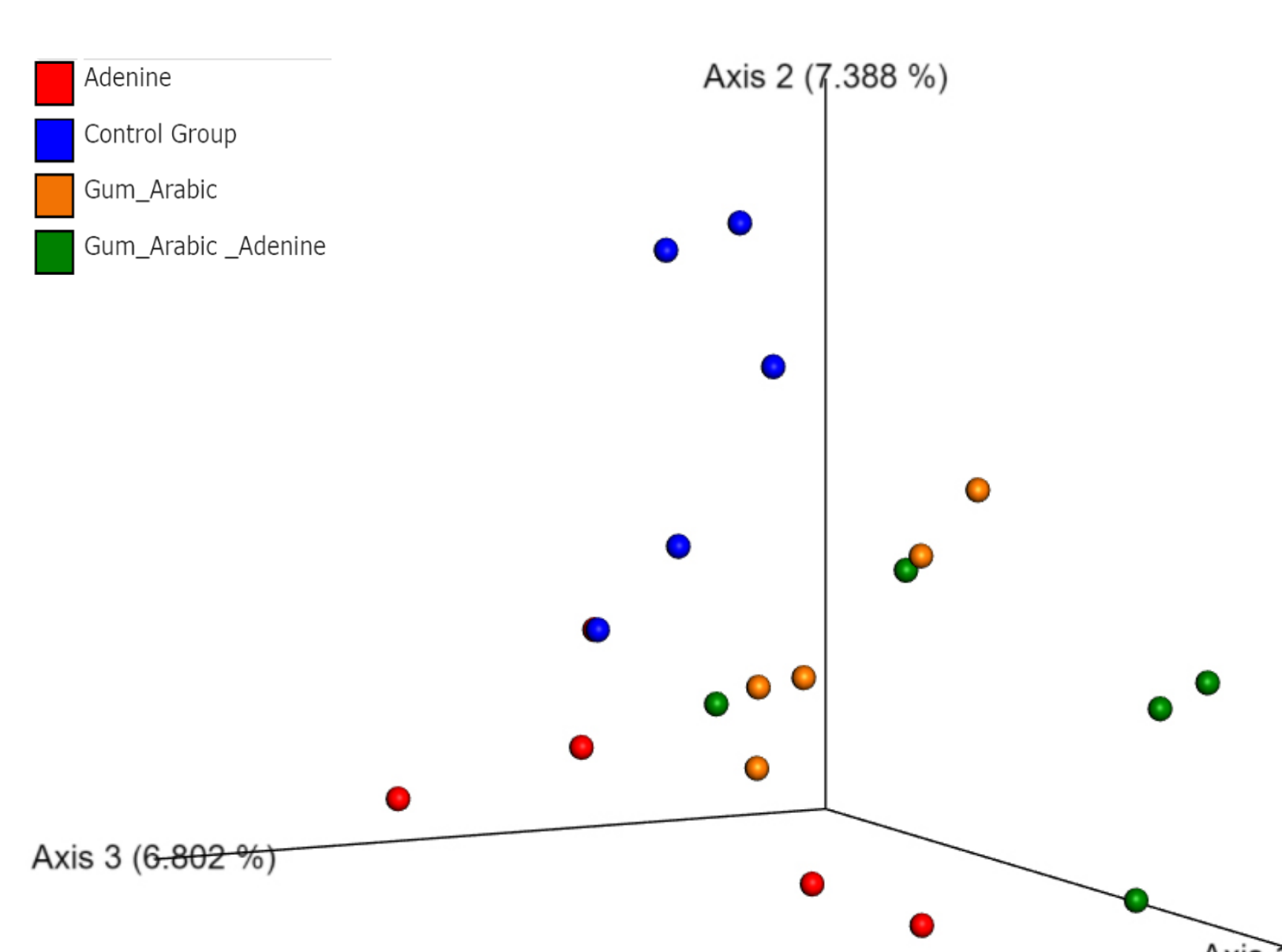

Beta diversity analysis in the colon shows a significant difference between the microbial populations in the control group and CKD group (p-value $<0.05$ ) .

Beta diversity analysis in the duodenum.

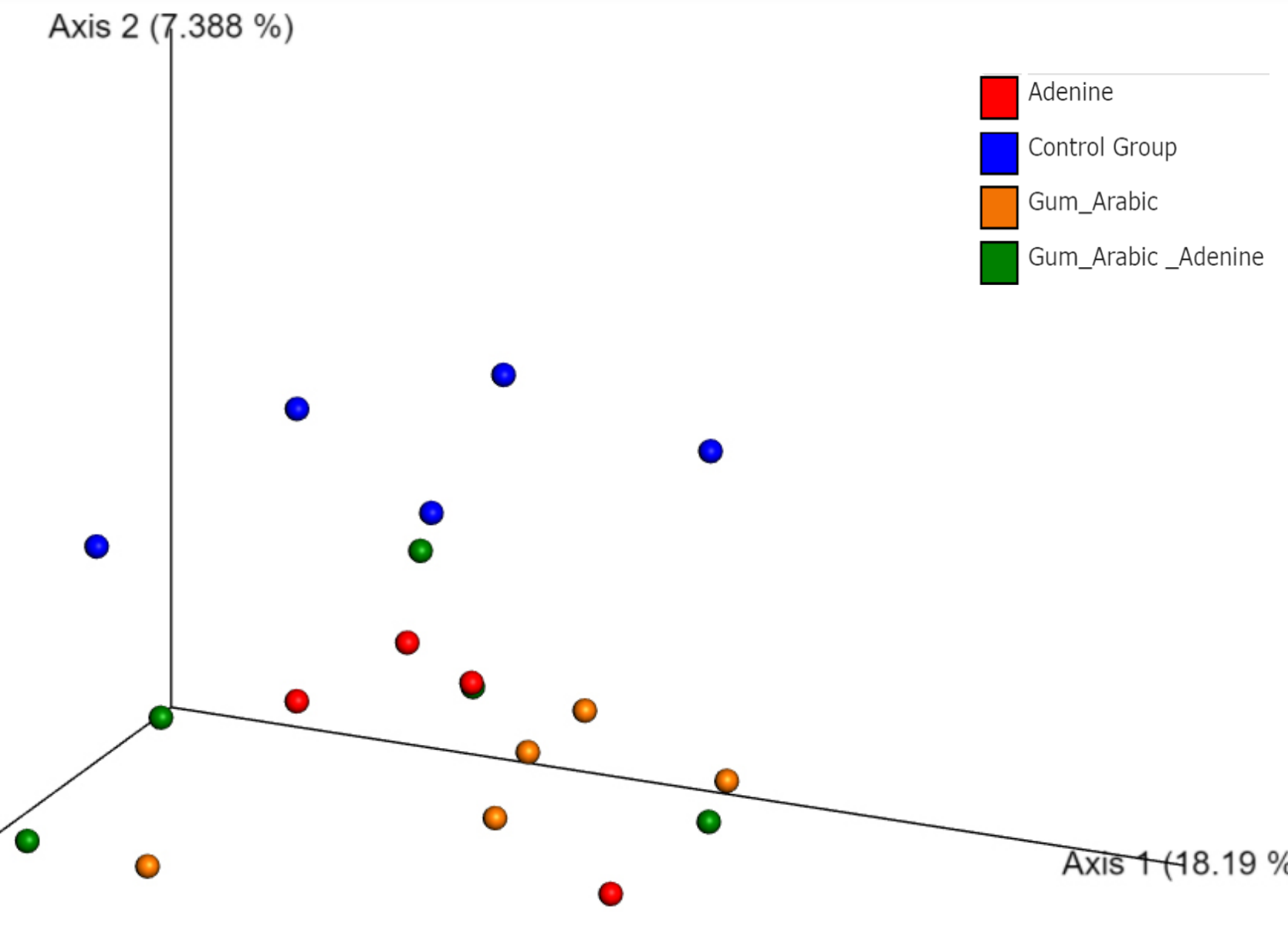

Beta diversity analysis in the duodenum shows a significant difference between the microbial populations in the control group and CKD group ( $\mathrm{p}$-value $<0.05$ )

\section{CONCLUSION}

In conclusion, CKD is associated with marked dysbiosis in the gut microbiome. GA has an effect in the gut microbiome of healthy and CKD rats. However, this effect is not well understood and further studies are needed.

\section{ACKNOWLEDGEMENTS}

We would like to express our gratitude to our supervisor, Dr. Maha Al Asmakh, our mentors Dr. Muhammed Sohail and Ms. Ola Al Jammal and to our collaborators Dr. Badreldin and Mr. Mohammed Ashique in Sultan Qaboos University. We would also like to thank the BRC for allowing us to use their laboratories.

\section{REFERENCES}

In the caecum, the diversity of the microbial population was significantly increased in GA group and treated CKD group. In the duodenum, the microbial diversity was markedly increased in the CKD group and decreased in the GA group and treated CKD group. In the colon, the microbial diversity was markedly decreased in the CKD group.
1. Nasir, O. (2013). Renal and extrarenal effects of gum arabic ( Acacia senegal )-what can be learned from animal experiments? Kidney Blood Press Res, 37(4-5), 269-279. doi:10.1159/000350152

2. Ramezani, A., \& Raj, D. S. (2014). The gut microbiome, kidney disease, and targeted interventions. J Am Soc Nephrol, 25(4), 657-670. doi:10.1681/ASN.2013080905 